\title{
Laparoscopic Groin Hernia Repair with Fixation through Transabdominal Polyprolene Stitches: A Randomized Prospective Study
}

\author{
Mohammed Said Ghali MD MRCS; Samy Gamil Akhnoukh MD; \\ Hany Rafik $M D$.
}

Department of General Surgery, Ain Shams University, Cairo, Egypt.

Background: Laparoscopic repair of inguinal hernias is associated with less postoperative pain and a faster rehabilitation, but its high cost was a changeling matter.

Patients and methods: We performed a randomized prospective study in Ain Shams University hospitals form July 2012 and April 2014 in which 50 male patients with primary unilateral or bilateral inguinal hernia were treated by laparoscopic transabdominal preperitoneal repair (TAPP) and mesh fixation through transabdominal polyprolene sutures. We recorded information about operative details, operative time, intraoperative complications, postoperative recovery, postoperative complications, and long term follow-up for one year after surgery.

Results: No major intra or postoperative complications had occurred, only 2 patients were converted to open Lichtenstein's repair. Mean operative time was significantly accepted. Hospital stay, recovery period were excellent. Only one patient had unacceptable postoperative pain which decreased over a year during his follow up. After a 1 year follow up period, only in single case of recurrence has been diagnosed.

Conclusion: Laparoscopic transabdominal preperitoneal repair (TAPP) and mesh fixation through transabdominal polyprolene stitches when efficiently experienced and mastered is an accepted and excellent surgical choice for inguinal hernia repair because of its cost effectiveness and less incidence of chronic pain syndrome.

Key words: Inguinal hernia, laparoscopic transabdominal preperitoneal repair (TAPP), transabdominal polyprolene stitches

\section{Introduction:}

Repair of inguinal hernia is one of the commonest surgical procedures performed world wide. ${ }^{1}$

Over the past 20 years, several hernia repair techniques have been introduced.

The ideal treatment to inguinal hernia should have the least per- and post operative morbidity; it should be the least traumatic as regards the requested type of anesthesia. ${ }^{2}$ It should offer the shortest hospital stay, the earliest recovery period and the least post-operative pain; it should be the least expensive and more importantly, it should have the least recurrence rates. The chosen technique should be also the easiest to learn and to perform.

The introduction of the Lichtenstein's tension-free repair has greatly decreased recurrence rates. Furthermore, it is a relatively easy to learn technique with no serious complications. ${ }^{3}$

Laparoscopic inguinal hernioplasty was first described by Ger ${ }^{4}$ in the early 1990s. Thereafter, laparoscopic repair of inguinal hernia has undergone many changes. ${ }^{5}$

The most widely accepted laparoscopic techniques nowadays are the transabdominal preperitoneal (TAPP) repair that was introduced by Arregui 6 and $\operatorname{Dion}^{7}$ in the early 1990s and the total extraperitoneal (TEP) repair introduced by Phillips $^{8}$ and 
McKernan $^{9}$ in 1993. Both techniques evolved from Stoppa's concept of preperitoneal reinforcement of fascia transversalis over myopectineal orifice by a prosthetic mesh. ${ }^{10}$

The development of the laparoscopic hernia procedure has been accompanied by a continuing search for mesh placement alternatives without mechanical fixation, ${ }^{11}$ including attempts to use fibrin glue. ${ }^{12}$

The potential complications related to fixation of mesh prosthesis using staples (especially postoperative neuralgia) prompted us to conduct this randomized prospective study and the use of this new technique of mesh fixation, through transabdominal polyprolene stitches.

\section{Patients and methods:}

This prospective randomized study was conducted in Ain Shams University hospitals form July 2012 and April 2014. A total of 50 male patients, $18-60$ years old with clinically diagnosed primary direct or indirect inguinal hernia ( 5 of them were having the hernia bilateral) were eligible for tension free laparoscopic repair using transabdominal preperitoneal approach (TAPP) with mesh fixation by transabdominal polyprolene stitches.

Four exclusion criteria for the participation were defined: (1) patients with huge, irreducible, or complicated hernia, (2) elderly patients with comorbidity (patients in American Society of Anesthesiologists "ASA" class 3, 4, and 5) where it was preferable to perform surgery with intravenous sedation and local anesthesia, (3) patients who had extensive lower abdominal or pelvic surgery in the past which would have made the laparoscopic dissection difficult or even impossible, and (4) patients' choice for a nonlaparoscopic operative approach.

\section{Preoperative Preparation:}

All patients were informed that conversion to open surgery might be required and a written consent was taken. All patients were asked to micturate before the operation. They all received prophylactic $3^{\text {rd }}$ generation cephalosporin.

\section{Operative techniques:}

A Veress needle was used to induce pneumoperitoneum at the umbilical site and then was replaced by one $10-12 \mathrm{~mm}$ optical trocar. A $5 \mathrm{~mm}$ trocar was positioned unilaterally on the umbilical line and a 10$\mathrm{mm}$ trocar was positioned in the iliac fossa contralateral to the hernia. In the presence of a bilateral hernia, both umbilical trocars were positioned bilaterally in the iliac fossa Figure (1). An incision of the wall of the peritoneum was made starting at the level of the superior margin of the internal inguinal ring at the level of the epigastric vessels Figure (2). It was dissected medially up to the residue of the umbilical artery and laterally for 3 to $4 \mathrm{~cm}$ past the inguinal ring for a total length of 7 to $8 \mathrm{~cm}$. In the presence of direct hernias, the hernial sac was directly isolated and reduced. In the case of indirect hernias, the preperitoneal parapubic adipose tissue was carefully dissected medially to expose the horizontal pubic ramus and Cooper's ligament. Accurate dissection of the preperitoneal retrovesical tissue permits easier positioning of the mesh. The internal inguinal ring was then explored, isolating and reducing the hernial sac; this maneuver was performed to reveal the presence of perihernial lipomas, which could then be removed.

Once the spermatic cord had been freed from the peritoneal wall, the prosthesis was positioned. A monofilament polypropylene mesh with large pores was used. The mesh was cut to a dimension of $10-15 \mathrm{~cm}$ and 2 separate $2 / 0$ polyprolene stitches with straight needle transfixing the abdominal wall from outside to inside Figure (3) then dragged to out side the abdomen through a $10-\mathrm{mm}$ trocar then we tie the 2 polyprolene stitches to the center of the mesh separately and $2 \mathrm{~cm}$ away from the lateral borders of the mesh then we drag the mesh to inside the abdomen with the help of polyprolene stitch and placed in the preperitoneal space such that it was in medial contact with the paravesical area, covering Cooper's ligament, resting on the inguinal region, and extending laterally over the epigastric vessels. At the end we tie the 
prolene stitch over the skin of inguinal region Figures $(4,5)$.

The peritoneal flaps were then closed using small, continuous, resorbable $2 / 0$ sutures or by using surgical clips Figure (6).

In patients with bilateral hernias, the same procedures were performed sequentially to repair the hernia on the other side (generally smaller).

\section{Post- operative care:}

During the period of hospital stay, all patients were given diclofenac sodium (75 $\mathrm{mg}$ IM /12 hours) as well as 3rd generation cephalosporin (1 gm IV /12 hours).

The time of hospital discharge was depending on two factors: 1) absence of serious operative and post operative complications. 2) good response to the injectable analgesic used in our protocol with no further need for other extra-analgesics. The medication described was the same for all patients at time of hospital discharge: Amoxicillin / clavulanic acid (2gm/day) for 1 week and diclofenac potassium (50mg tablet) taken only on demand for 1 week. The severity of pain during the 1st post-operative week was assessed by the dose of diclofenac potassium calculated by the number of tablets $(50 \mathrm{mg})$ taken every day during that week.

\section{Data collection and follow up:}

\section{Preoperative parameters:}

The following parameters were recorded: Age, body mass index (BMI), type, ASA, and duration and size of hernia. All patients were asked before surgery to rate their pain or discomfort on a visual analog scale (VAS) from 0 (none) to 10 (worst).

\section{Intraoperative parameters:}

The following parameters were recorded: Type of hernia, the operation time, (skin to skin) in minutes, operative complications such as, bowel perforation, urinary bladder injury, spermatic cord or vas injury, vascular injury and bleeding, peritoneal defects, and conversion of TAPP technique to open repair. The cost of the procedure was also recorded.

\section{Postoperative parameters:}

Post-operatively all early potential complications, such as urinary retention, hematoma, seroma, bleeding and wound infection were assessed and documented.

\section{Follow up measures:}

All patients were encouraged to return to work and normal activities as soon as possible. Time to full recovery was noted by the patient and was recorded. It was defined as the number of days between the day of surgery and the first day a patient was able to perform full daily activities. Time of return to work was also recorded.

\section{A) Primary outcome:}

The primary outcome of the trial was early and late postoperative neuralgia, defined by the presence of intermittent hyperesthesia, burning sensation and/or jabbing pain in the ipsilateral inguinal area nerves (genitofemoral nerve, lateral cutaneous femoral nerve, ilioinguinal and iliohypogastric nerves).

The patients were asked to assess the pain severity and its site every day for the first week and to record the number of diclofenac potassium tablets $(50 \mathrm{mg})$ taken every day during the first post operative week.

Postoperatively, patients were examined at 1, 3, 6 and 12 months to determine the presence or absence of postoperative neuralgia using the visual analog scale (VAS).

Patients who experienced groin, scrotal, or thigh pain at 6 months after surgery were considered to have chronic pain. These patients were asked to describe the character, site, and severity of the pain. Patients were also asked about whether pain was affecting their normal daily and / or work / sporting activity.

\section{B) Secondary outcomes:}

The patients were asked to return to the outpatient clinic at one week and then at 1 , 3, 6 and 12 months for a standardized history taking and physical examination of the wound, testis, nerve sensation, port-site to detect wound tenderness, wound/mesh infections, port-site hernia, hydrocele, orchitis, testicular 
atrophy, sensory loss, and most importantly recurrence which was defined as a clinically detectable reducible swelling in the treated groin. In doubtful cases, ultrasonography of the groin was performed.

\section{Results:}

\section{Patients flow:}

50 male patients with inguinal hernia (5 of them were bilateral) were included in the study. Their mean age was $32 \pm 10$ (range: 18-60) years.

\section{Preoperative parameters:}

The demographics of the patients regarding age, BMI, ASA classification, and duration and size of hernia as shown in Table (1).

\section{Intraoperative parameters:}

Intraoperative data are shown in Table (2). Operative time was around 74 minutes (range 62-109 minutes).

Injury of the inferior epigastric vessels occurred in 2 cases (4\%); the injured vessel has been clearly identified and ligated by clips in 1 patient while in the remaining patient bleeding was not controlled and it obscured the field completely; conversion to open repair and controlling of the bleeder has been therefore performed.

Large peritoneal tears occurred in 4 cases $(8 \%)$. Failure of closure of the peritoneal defect occurred in 1 of them who was converted to open Lichtenstein's technique.

A small intestinal injury (small electrocautery burn not perforating the mucosa) occurred in 1 patient and has been primarily sutured laparoscopically and the repair procedure has been successfully continued without conversion to open repair. This patient passed a nice postoperative period and has been discharged on the 5th day after the operation. No bladder, or cord injury were recorded in any patient.

\section{Early Post-operative parameters:}

Early post-operative complications are shown in Table (3). Urinary retention occurred in 2 patients (4\%), the condition was transient and was managed by urinary catheterization.

Umbilical port site seroma occurred in 3 patients $(6 \%), 2$ of them were complicated by wound infection, one of them was complicated by umbilical granuloma that necessitated surgical excision and curettage performed 42 days after the repair. Scrotal hematoma occurred in 4 patients (8\%) and they were successfully managed conservatively.

The mean hospital stay was 2 days (range 1-5days) with $80 \%$ of patients were discharged after 24 hours of the procedure. The mean time to return to normal activities was 7 days (range 5-15 days); and the mean time to return to work was 12 days (range 1025 days).

\section{Long term follow up:}

Long term complications are shown in Table (4). During a period of 1 year follow up, recurrence occurred in 1 patient $(2 \%)$, this patient had undergone open Lichtenstein's repair.

A single case of post repair moderate hydrocele occurred in one patient and it was planned to treat him surgically. No cases of postoperative orchitis, testicular atrophy, port-site hernia, internal hernia or postoperative adhesive intestinal obstruction have been recorded in any patient after a 1 year of follow up period.

\section{Evaluation of perioperative and long-term postoperative pain:}

This is one of our primary outcomes of the study. Average pain score preoperatively on the VAS was (5.5) and averaged (3.5) immediately postoperatively and at time of hospital discharge with only 1 patient required extranalgesia in the first postoperative week. VAS decreased to (0.5) at 1-month follow-up with only 1 patient rating his pain (3). And at one year follow up, all patients were free of pain.

\section{Patients' satisfaction:}

The patient satisfaction at 1-month followup was categorized as either really satisfied with $80 \%(n=40 / 50)$, satisfied with $18 \%$ 


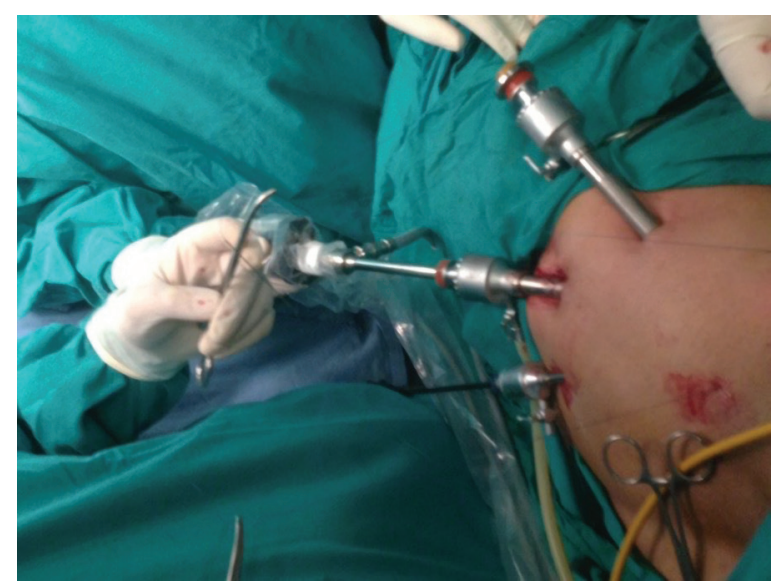

Figure (1): Trocars positioning

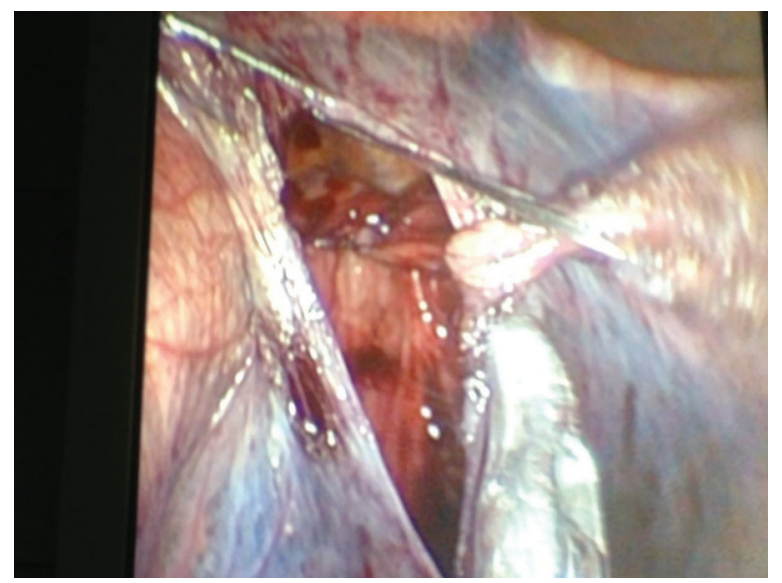

Figure (3): A polyprolene suture on a straight needle passing transabdominal

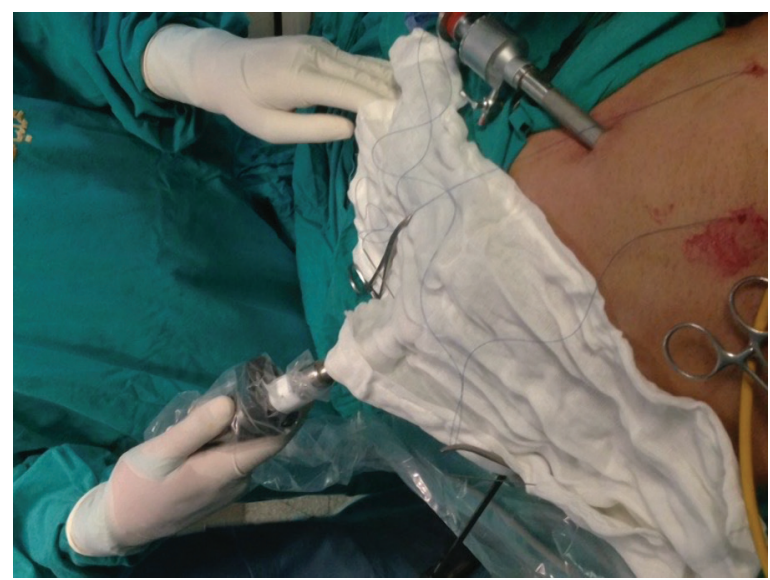

Figure (5): Transabdominal mesh fixation

$(\mathrm{n}=9 / 50)$, and not really Satisfied with $2 \%$ $(n=1 / 50)$.

The patient who was not really satisfied had persistent postoperative pain at 1-month follow-up with a VAS pain of (3), which decreased to (0) at 1-year follow-up

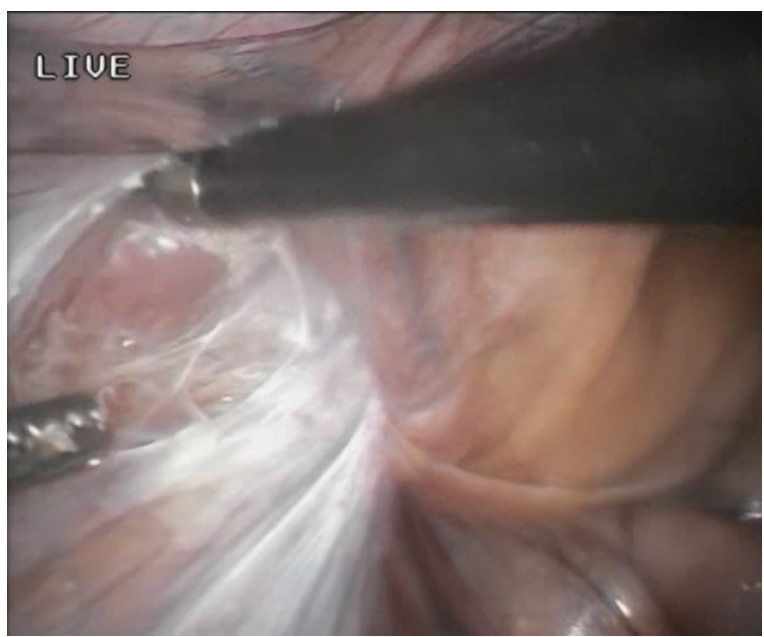

Figure (2): Peritoneum is incised

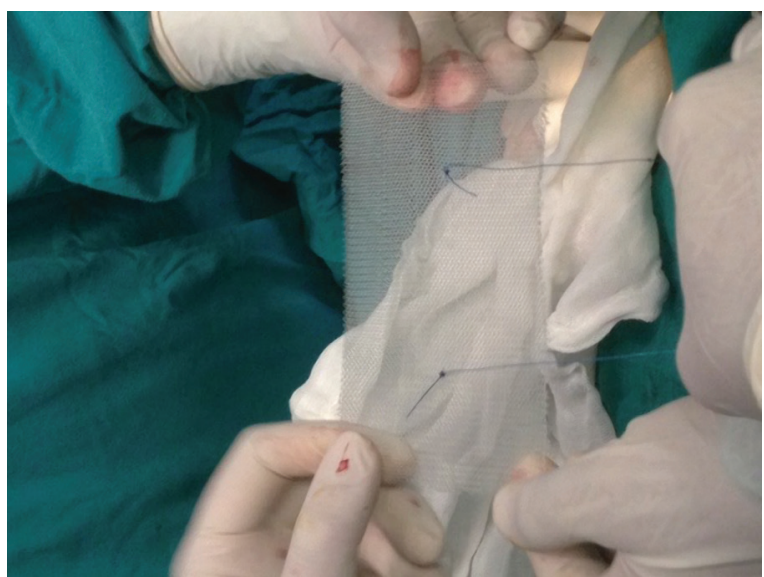

Figure (4): Transabdominal mesh fixation

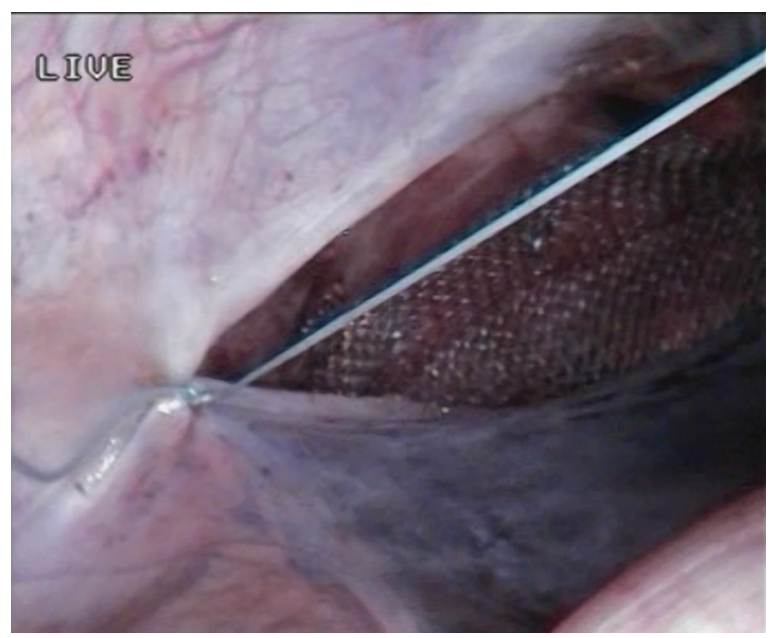

Figure (6): Closure of the peritoneum

evaluation.

Cost evaluation of TAPP using our technique versus open Lichtenstein's hernioplasty \& other TAPP techniques:

This is one of our primary outcomes 
Table (1): Baseline characteristics of the patients according to treatment group.

\begin{tabular}{ll}
\hline Variable & \\
\hline Mean Age (years) & $32 \pm 10$ \\
BMI & 30.6 \\
ASA classification: & \\
$\quad$ I & $42 / 50$ \\
$\quad$ II & $8 / 50$ \\
Duration of hernia: & \\
$\quad<2$ months & $8 / 50$ \\
$\quad 2-12$ months & $20 / 50$ \\
$\quad>12$ months & $22 / 50$ \\
Size of hernia: & \\
• Bulges only when straining & $25 / 50$ \\
- Visible bulge when standing but not extending to scrotum & $17 / 50$ \\
- Scrotal but not huge & $8 / 50$ \\
Unilateral or bilateral: & \\
$\quad$ Unilateral & $45 / 50$ \\
$\quad$ Bilateral & $5 / 50$ \\
\hline
\end{tabular}

Table (2): Characteristics of surgery and operative complications

\begin{tabular}{ll}
\hline Variable & \\
\hline Mean operative time (min) & 74 \\
Type of hernia (\%) & 22,5 \\
- Direct & 65 \\
- Indirect & 12.5 \\
- Dual & \\
Operative complications (no): & 2 \\
- Conversion to open technique & 0 \\
- Vas deferens injury & 1 \\
- Bowel injury & 0 \\
- Urinary bladder injury & 2 \\
- Inferior epigastric v. Injury & 0 \\
- Major vessel injury & \\
\hline
\end{tabular}

of the study. Comprehensive evaluation revealed that institutional costs for operation room use, laboratory and other diagnostics, hospital postoperative care as well as many operation related costs, for example, gloves, disinfection, urinary catheter, steri-strips, gauze, etc, are similar or identical for our technique and open hernioplasty procedures.

Finally, the cost difference between the
2 procedures comes down to the difference in price of the mesh used, cost for an insufflation tubing and use of $\mathrm{CO} 2$ for the laparoscopic hernia repair and additional sutures used for the open procedure. The total cost of all nonreusable items such as drapes, suture, mesh, gowns, gloves, etc, for a 1-side TAPP repair at our ambulatory surgery facility is 550 L.E. (Livre egyptienne). Total 
Table (3): Early post-operative complications

\begin{tabular}{ll}
\hline Variable & No \\
Related death & 0 \\
Urinary retention & 2 \\
Wound infection & 2 \\
Seroma & 3 \\
Scrotal hematoma & 4 \\
\hline
\end{tabular}

Table (4): Long term complications

\begin{tabular}{ll}
\hline Variable & No \\
\hline Recurrence & 1 \\
Hydrocele & 1 \\
Port-site hernia & 0 \\
Umbilical granuloma & 1 \\
Internal hernia & 0 \\
Intestinal obstruction & 0 \\
Orchitis & 0 \\
Testicular atrophy & 0 \\
\hline
\end{tabular}

costs of disposable items for a 1-sided open hernioplasty is 250 L.E., the difference between the 2 being mainly the cost for material needed for a laparoscopic procedure.

The cost difference between our new technique of fixation using transabdominal polyprolene stitches and other way of fixation in TAPP using fibrin Glue (Tissucol) or staples for mesh fixation is that our new technique saves about 500 L.E.

\section{Discussion:}

This randomized clinical study evaluates a new technique for mesh fixation during TAPP repair of inguinal hernia through transabdominal polyprolene stitches.

In our study we have chosen TAPP technique rather than total extraperitoneal technique (TEP) because TAPP operation is easier to learn and master, ${ }^{13}$ and does not suffer the same risks of preperitoneal bleeding and oozing as can be seen in the TEP procedure. ${ }^{14}$ Furthermore, TAPP gives better view of the inguinal anatomy1. Another advantage of TAPP approach is that it allows inspection of both groins, and identification of asymptomatic defects. ${ }^{15}$
In our study the TAPP operations caused less pain in the early post-operative period, leading to earlier mobilization, shorter hospital stay and earlier return to work than Lichtenstein's repair. This is mostly attributed to smaller incisions, the absence of muscle dissection during TAPP technique and hence rapid healing. Furthermore, laparoscopic TAPP is associated with greater patient satisfaction and better cosmetic results than its open counterpart. These results are in line with many randomized series. $2,16,17,18,19$

The rate of conversion from TAPP to open Lichtenstein technique was 2/50. This is directly related to the learning curve as conversion occurred in 2 patients of the first 15 operations only. This rate was nearly the same as in other studies. ${ }^{5}$

In our study, only one case of minor intestinal injury was reported, injury of inferior epigastric vessels occurred in 2 patients, no other serious operative or lifethreatening complications occurred. Though other studies have reported that intraoperative, immediate postoperative complications occur more frequent in the laparoscopic repair group than in the open repair group. 16,20,21 
The results of our study showed that the operative time is obviously longer in TAPP procedure when compared to Lichtenstein procedure. This is clearly attributed to the learning curve as proved by the marked decrease in operative time in the last twenty repairs performed laparoscopically and also a marked decrease in the mean operative time compared to another study done in Ain Shams university hospitals previously. ${ }^{22}$ These results are in line with that obtained by many other trials. ${ }^{23,24}$ However, in the study carried by David, 25 the mean operating time for a unilateral hernia managed by TAPP was only 25 minutes. Another large series of 12678 cases of TAPP hernia repair showed a mean operative time of 40 minutes. ${ }^{26}$ Furthermore, another trial carried by Roseano and others ${ }^{2}$ reported shorter operative time in the laparoscopic group than in Lichtenstein's group.

Chronic pain syndrome remains one of the challenging problems of inguinal hernia repair and this problem was one of the major aims of this study. The pain may be explained by: 1) Mesh induced fibrosis. 2) Nerve entrapment by a staple. 3) Extensive dissection of the sac from cord structures. 4) Injury of the genital branch of genito- femoral nerve during extensive skeletonization of the cord. 5) Compression or irritation of the nerve secondary to fibrosis around the cord.

6) Direct nerve cutting or cauterization.

In laparoscopic hernia repair, several different anchoring techniques have been used and recommended since the early 1990s such as staples, coils etc, but any mechanical anchoring carries the risk of causing temporary or permanent pain or discomfort or can even damage sensitive structures such as nearby nerves and vessels. ${ }^{27}$ To avoid such risks, alternative strategies with fixation using transabdominal polyprolene stitches which prevents migration of the mesh and eliminates the use of additional anchoring techniques in our experience.

Chronic inguinal pain, its cause, and treatment have become significant issues in long-term outcome studies. Therefore, the characteristics of the mesh fixation using transabdominal plyproprolene stitches must be taken into consideration to avoid nerve entrapment, Thus far in follow-up of our experience with fixation using transabdominal polyproprolene stitches, long-term pain is minimal to none and patient satisfaction is high.

A number of studies ${ }^{3,22,28}$ reported a higher incidence of chronic pain after open repair of inguinal hernia. One hypothesis is that the dissection and fixation in the Lichtenstein's method is more extensive than TAPP, which could increase the risk of nerve injuries leading to pain and /or sensory loss.

In our study we also reported that chronic pain is less frequent when done by our technique in comparison to another study done in Ain Shams university hospitals when mesh fixation was done by staples through TAAP approach. ${ }^{22}$

The cost of the procedure remains also one of the challenging problems of laparoscopic inguinal hernia repair and this problem was also one of the major aims of this study. One of the most important drawbacks of TAPP is its higher cost when compared to Lichtenstein's repair. Many authors agree about this point. ${ }^{23,29}$ Although TAPP technique resulted in shorter hospital stay and shorter time to return to work, but this is not enough to compensate for the increased cost. ${ }^{2}$

In an attempt to evaluate the true cost to the facility, we looked at the material cost to the facility of the disposable items used and not the "charges" which we all know are different. To truly assess the difference in cost between procedures, it is also important that comparison should be made within the same facility and not between different facilities.

With a difference of only 250 L.E more than open surgery and 500 L.E. Less than TAPP with mesh fixation by staples or fibrin glue, the cost gap between laparoscopic and open hernia repair and between our technique of fixation to the other techniques is well accepted.

Recurrence is the most important parameter in the evaluation of inguinal hernia repair. However, meta-analysis of randomized controlled trials 2,30 has demonstrated 
that there was no significant difference in recurrence rate when TAPP was compared with open hernioplasty.

In our study recurrence occurred only in one patient. This is mostly attributed to technical errors such as inability to dissect enough preperitoneal space for mesh placement or due to rolling of the mesh. The learning curve is another important factor; this is proved by our results that showed that the single patient complicated by recurrence was performed in the first 3 months of our study. This recurrence rate is similar when compared to other studies. ${ }^{31,32}$

\section{Conclusion :}

Laparoscopic groin hernia repair with fixation through transabdominal plyprolene stitches has been shown to have excellent longterm results regarding pain, low recurrence rate at only marginally increased costs above open hernia procedures and decreased costs below other anchoring devices.

With a low recurrence rate, acceptable cost and low incidence of long-term chronic pain, laparoscopic groin hernia repair with fixation through transabdominal polyprolene stitches is the procedure of choice for our patients at present time.

\section{Reference:}

1- Kriplani A.K, Shyam S, Daipayan G: Laparoscopic Trans Adominal Pre-Peritoneal (TAPP) Repair of Inguinal Hernia. In: Comprehensive Laparoscopic Surgery. Sagar Printer, New Delhi 2007; 119-130.

2- Roseano M, Ressetta G, Pozzetto B, Babich F, De Manzini N: The treatment of inguinal hernia in the elderly: Open technique or laparoscopic approach? Acta Biomed 2005; 76: 52-55.

3- Grant A: Mesh compared with non mesh methods of open groin hernia repair: Systematic review of randomized controlled trials. Br J Surg 2000; 87: 854-859.

4- Ger R: The laparoscopic management of groin hernias. Contemp Surg 1991; 39(4): 15-19.

5- David W, Caron P, Neil S, et al: Five year follow-up of patients undergoing laparoscopic or open groin hernia repair: A randomized controlled trial. Ann Surg 2002;
235(3): 333-337.

6- Arregui ME, Davis CJ, Yucel O, et al: Laparoscopic mesh repair of inguinal hernia using a preperitoneal approach: A preliminary report. Surg Laparosc Endosc 1992; 2: 53-58.

7- Dion YM, Morin J: Laparoscopic inguinal herniorraphy. Can J Surg 1992; 35: 209-212.

8- Philips EH, Carroll BJ, Fallas MF: Laparoscopic preperitoneal inguinal hernia repair without preperitoneal incision: Technique and early results. Surg Endosc 1993; 7: 159-162.

9- McKernan JB, Laws HL: Laparoscopic repair of inguinal hernias using a totally extraperitoneal prosthetic approach. Surg Endosc 1993; 7: 26-28.

10- Snehal F: Laparoscopic versus open repair of inguinal hernia. World J Laparosc Surg 2008; 1(1): 41-48.

11- Ferzli GS, Frezza EE, Pecoraro AM JR, et al: Prospective randomized study of stapled versus unstapled mesh in a laparoscopic preperitoneal inguinal hernia repair. $\mathrm{J} \mathrm{Am}$ Coll Surg 1999; 188: 461-465.

12- Schwab R, Willms A, Kroger A, Becker HP: Less chronic pain following mesh fixation using fibrin sealant in TEP inguinal hernia repair. Hernia 2006; 10(3): 272-277.

13- David S: Laparoscopic transabdominal preperitoneal (TAPP) repair of groin hernia: One surgeon's experience of a developing technique. Ann R Coll Surg Engl 2002; 84: 393-398.

14- Thumbe VJ, Evans DS: To repair or not to repair incidental defects found on laparoscopic repair of groin hernia. Early results of a randomized control trial. Surg Endosc 2001; 15: 47-49.

15- David W, Caron P, Neil S, et al: FiveYear Follow-Up Of Patients Undergoing Laparoscopic Or Open Groin Hernia Repair: A randomized controlled trial. Ann Surg 2002; 235(3): 333-337.

16- Leigh N, Anita G, Olga J, et al: Open Mesh versus Laparoscopic Mesh Repair of Inguinal Hernia. $N$ ENGL $J$ MED 2004; 350 : 1819-1827.

17- Douek M, Smith G, Stoker D, et al: Prospective randomized controlled trial of laparoscopic versus open inguinal hernia mesh repair : Five year follow up. BMJ 2003; 326: 1012-1013.

18- Franneby U, Sandblom G, Nordin P, et al: Risk Factors for Long-Term Pain After Hernia Surgery. Ann Surg 2006; 244: 212-219. 
19- Grant AM, Scott NW, O’Dwyer PJ: Fiveyear follow-up of a randomized trial to assess pain and numbness after laparoscopic or open repair of groin hernia. Br J Surg. 2004; 91: $1570-1574$.

20- Medical Research Council Laparoscopic Groin Hernia Trial Group: Laparoscopic versus open repair of groin hernia: A randomized comparison. Lancet 1999; 354: 185-190.

21- McCormack K, Scott NW, Go PM, Ross $\mathrm{S}$, et al: Laparoscopic techniques versus open techniques for inguinal hernia repair. Cochrane Database Syst Rev 2003; 1: CD001785.

22- Nafei A, Salib WF, Ali AH, et al: Is laparoscopic transabdominal preperitoneal repair of inguinal hernia a good alternative to Lichtenstein's Repair? A Randomized Prospective Trial. Ain Shams J Surg 2010; 3(2): 216-229.

23- Johansson B, Hallerback B, Glise H, et al: Laparoscopic Mesh Versus Open Preperitoneal Mesh Versus Conventional Technique for Inguinal Hernia Repair. Ann Surg 1999; 230: 225-231.

24- SabanciU, Ogun I, Candemir G: Laparoscopic inguinal hernia repair results of a military hospital in Turkey. Balkan Military Medical Review 2007; 10:132-135.

25- David S: Laparoscopic transabdominal preperitoneal (TAPP) repair of groin hernia: One surgeon's experience of a developing technique. Ann R Coll Surg Engl 2002; 84:
393-398.

26- Bittner R, Leibl BJ, Jager C, et al: TAPPStuttgart technique and result of large single center series. J of Min Access Surg 2006; 2(3): 158-159.

27- Bueno J, Serralta A, Planells M, et al: Inguinodynia after two inguinal herniorrhaphy methods. Surg Laparosc Endosc Percutan Tech 2004; 14: 210-214.

28- Douek M, Smith G, Stoker D, et al: Prospective randomized controlled trial of laparoscopic versus open inguinal hernia mesh repair: Five year follow up. BMJ 2003; 326: 1012-1013.

29- Medical Research Council Laparoscopic Groin Hernia Trial Group: Cost utility analysis of open versus laparoscopic groin hernia repair: Results from a multicenter randomized clinical trial. Br J Surg 2001; 88: 653-661.

30- Grant A: Laparoscopic versus open groin hernia repair: Meta-analysis of randomized trials based on individual patient data. The EU Hernia Trialists Collaboration. Hernia 2002; 6: 2-10.

31- Moreno-Egea A, Aguayo JL, Canteras M: Intraoperative and postoperative complications of totally extraperitoneal laparoscopic inguinal hernioplasty. Surg Laparosc Endosc Percutan Tech 2000; 10: 30-33.

32- Voyles CR: Outcomes analysis for groin hernia repairs. Surg Clin North Am 2003; 83: 1279-1287. 